\title{
Motor assessment in Parkinson`s disease
}

\author{
Józef A Opara', Andrzej Małecki', Elżbieta Małecka', Teresa Socha' \\ 1 Jerzy Kukuczka Academy of Physical Education in Katowice, Poland \\ Opara J A, Małecki A, Małecka E, Socha T. Motor assessment in Parkinson`s disease. Ann Agric Environ Med. 2017 ; $24(3)$ : 411-415. \\ doi: $10.5604 / 12321966.1232774$
}

\begin{abstract}
Parkinson's disease (PD) is one of most disabling disorders of the central nervous system. The motor symptoms of Parkinson's disease: shaking, rigidity, slowness of movement, postural instability and difficulty with walking and gait, are difficult to measure. When disease symptoms become more pronounced, the patient experiences difficulties with hand function and walking, and is prone to falls. Baseline motor impairment and cognitive impairment are probable predictors of more rapid motor decline and disability. An additional difficulty is the variability of the symptoms caused by adverse effects of drugs, especially levodopa.

Motor assessment of Parkinson's Disease can be divided into clinimetrics, assessment of balance and posture, arm and hand function, and gait/walking. These are many clinimetric scales used in Parkinson`s Disease, the most popular being the Hoehn and Yahr stages of progression of the disease and Unified Parkinson's Disease Rating Scale. Balance and posture can be assessed by clinimetric scales like the Berg BS, Tinetti, Brunel BA, and Timed Up and Go Test, or measured by posturometric platforms. Among skill tests, the best known are: the Purdue Pegboard Test, Nine-Hole Peg Test, Jebsen and Taylor test, PigTail Test, Frenchay Arm Test, Action Research Arm Test, Wolf FMT and Finger-Tapping Test. Among motricity scales, the most popular are: the Fugl-Meyer Motor Assessment Scale and Södring Motor Evaluation. Gait and walking can also be assessed quantitatively and qualitatively. Recently, the most popular is three-dimensional analysis of movement. This review article presents the current possibilities of motor assessment in Parkinson`s disease.
\end{abstract}

\section{Key words}

Parkinson`s disease, PD, balance, gait, posture, posturometry

\section{INTRODUCTION}

Parkinson's disease (PD) is a degenerative disorder of the central nervous system. The motor symptoms of Parkinson's disease result from the death of dopamine-generating cells in the substantia nigra, a region of the midbrain; the cause of this cell death is unknown. In the course of the disease, the most obvious symptoms are movement-related: tremor, rigidity, slowness of movement, postural instability and difficulty with walking and gait. The disease is more common in older people, with most cases occurring after the age of 50 . PD, which affects $2 \%$ of the population above 65 years of age and $4 \%$ of those over 85 years, is one of the most frequent causes of physical disability. When the disease symptoms become more pronounced, the patient experiences difficulties with hand function and walking, and prone to falls.

The principles of diagnose of PD were created in 1992 by Hughes et al., and developed in 1999 by Gelb et al. The clinical course of PD varies from patient-to-patient.

To predict the rate of motor decline and increasing disability in early PD, Marras et al. (2002) conducted a systematic review of English-language and French-language literature cited in the MEDLINE database (1966-2002). The results of 13 studies were summarized qualitatively. The study methods were highly variable, particularly regarding the choice of outcome measure. Baseline motor impairment and cognitive impairment are probable predictors of more rapid motor decline and disability. A lack of tremor at onset and older age both appear to be predictive of increasing disability, but conflicting results exist for their association with the rate of change of motor impairment. Family history

Address for correspondence: Józef A Opara, Jerzy Kukuczka Academy of Physical Education in Katowice

E-mail: jozefopara@wp.pl

Received: 9 July 2014; accepted: 3 November 2014; first published: February 2017 of PD does not appear to be prognostically important. The prognostic value of many other factors studied is uncertain owing to conflicting or unconfirmed results.

Clinimetrics in Parkinson's Disease. Clinimetrics in PD is a particularly difficult task. The basic symptoms of PD: bradykinesia, propulsion, retropulsion, lateropulsion, demarche à petit pas, lack of arms co-movements, problems with handwriting (micrograph), muscle rigidity, and tremor, can scarcely be assessed. An additional difficulty is the variability of the symptoms caused by adverse effects of drugs, especially levodopa.

The oldest scale for PD was published in 1967 by Margaret Hoehn and Melvin Yahr, as the result of observation of 856 patients (Hoehn \& Yahr, 1967; Opara, 1998 and 2012). It distinguishes five stages of injury and disability, defined as five stages of progression of the disease, from unilateral damage without disorders as stage $\mathrm{I}$, to stage $\mathrm{V}$, when the patient is confined in a wheelchair or bed. To-date, this scale is the most commonly used worldwide, especially in multicentre studies.

In 1987, Fahn and Elton headed a task force which developed the Unified Parkinson's Disease Rating Scale (UPDRS) which consists of six integral parts. These are:

I - the state of intellectual and mood disorders;

II - activities of daily living (separately for phase 'on' and 'off');

III - motor examination;

IV - complications of treatment;

$\mathrm{V}$ - stages of the disease;

VI - self-assessment of independence using the SchwabEngland Scale.

The first three areas and part of the fourth are assessed in a five-point scale ( $0-4$ points). In the third part (motor) 
UPDRS assesses the speech, facies, tremor in resting, tremor intentional, rigidity, rapid movements of the fingers, rapid hand movements, alternating movements, leg movements, getting up from a chair, posture, stability of posture, starting walking and bradykinesia. Scoring system:

0 point $=$ no involvement;

$1=$ detectable disorders;

2 = moderate disorders;

3 = considerable disorders;

$4=$ no function or severe disorders.

In 2008, Goetz et al., in the name of the Movement Disorder Society (MDS), published a modified UPDRS, called the MDS - sponsored revision of the Unified Parkinson's Disease Rating Scale, abbreviated to MDS-UPDRS. The revised MDS-UPDRS consists of 65 items, compared with the 55 of the original UPDRS, including 48 items in which the assessment ranges from $0-4$, and 7 options with answers 'yes' or 'no'. The 20 questions correspond to the patient or caregiver. In Part I, there are 13 items, in Part II - 13, in Parts III and Part IV - 33, and 6 positions. MDS-UPDRS showed high internal consistency (Cronbach's alpha $=0.79-0.93$ ) and high correlation with the original UPDRS (rho =.96). There was a strong structural reliability of the individual parts (comparative fit index $>0.90$ for each part). Therefore, it is recommended to calculate the total score for each of the four parts separately, instead of the total final score of all parts together. Despite the fact that the clinimetric usefulness of the MDS-UPDRS XP for scientific research has been confirmed, the old version of Hoehn \& Yahr scale and UPDRS is still in use.

In 2004, Marinus et al. described the Short Scale Evaluation Parkinson / Scale for Assessment Results (treatment) PD - Short Parkinson's Evaluation Scale (SPES) / Scales for Outcomes in Parkinson's disease (SCOP), a brief scale designed to assess motor function in PD patients. The scale (score of 0 - normal - to 3 - severe) estimates 21 parameters in the three sub-scales. These include: assessment of physical (motor evaluation), activities of daily living (ADL) and motor complications (motor complications). Studies have shown the usefulness of SPES / SCOPE, both in research and in clinical practice (Marinus et al., 2004; Martinez-Martin et al., 2008].

Balance and posture in PD. Some people suffering from PD experience disturbances with keeping balance and stable posture. Sometimes, they fall down which can be dangerous. Statistical data show that falls affect more than $50 \%$ of PD patients (Bloem et al., 2001; Opara et al., 2005). To assess the balance and control of posture, the Timed Up and Go Test (TUG) by Podsiadło and Richardson (1989) is commonly used; it measures the time needed for the patient to get up from a chair, going 10 feet, making the return and re-occupying the chair. In 2001, Morris et al. evaluated the suitability of TUG in detecting changes in mobility in patients with PD. The usefulness of TUG in PD has been confimed. In 2002, Siggeirsdóttir et al. found that the TUG test is the most reliable when the height of the chair seat ranges from $44-47 \mathrm{~cm}$.

For assessment of balance, the Berg Balance Scale (BBS, 1992), Tinetti Balance and Gait Assessment Tool (1987), Brunel Balance Assessment (BBA, 2002), and many other scales are used (Opara, 2012; Tinetti, 1986). The Functional Reach Test (FRT) is useful for identifying falls risk among individuals with PD (Behrman et al., 2002).
In 2009, Horak et al. published the Balance Evaluation Systems Test (Bestest) which consists of 36 parts, grouped into 6 systems:

1. biomechanical constraints;

2. stability limits/verticality;

3. anticipatory postural adjustments;

4. postural responses,

5. sensory orientation;

6. stability in gait.

In 2012, Franchignoni et al. published a shortened version of the test - MiniBESTest, modified in 2013 by King and Horak. The currently used version of MiniBESTest consists of 14 points, comprising four subscales with the point system $0-2$; maximum score -28 points.

In the $21^{\text {st }}$ century, posturometric and stabilometric evaluation has developed rapidly, and has also been used for evaluation in balance training [Stożek et al., 2003; van der Burg et al., 2006). Błaszczyk and Orawiec (2011) analyzed sway ratio (SR) in patients with PD and age-matched healthy subjects. The sway ratios were assessed based on centre of foot-pressure (CP) signals recorded in $55 \mathrm{PD}$ patients (Hoehn and Yahr: 1-3) and 55 age-matched healthy volunteers, while standing quietly with eyes open (EO), and then with eyes closed (EC). Complementing classical sway measure abnormalities, the SR exhibited a high discriminative power for all controlled factors: pathology, vision, and direction of sway. Both the anteroposterior (AP) and mediolateral (ML) sway ratios were significantly increased in $\mathrm{PD}$ patients, when compared to the control group.

In 2012, Song et al. stated that compared to healthy controls, persons with early PD altered their postural control strategies (shorter distance between the centre of pressure (COP) and the extrapolated centre of mass (COM) during the $90^{\circ}$ step turn. Persons with early PD appear to decrease their overall movement amplitude (i.e., COM displacement, velocity) suggesting that dynamic postural control during turning is altered even in the early stages of PD. In 2013, Słomka et al. stated that at least three 60 -s trials should be used when utilizing selected measures of centre of pressure (COP) in the rambling (RM) and trembling (TR) decomposition of a stabilogram.

Patients with PD demonstrate deficits in motor learning as well as bidirectional interference (the performance of one task concurrently interferes with the performance of another task) during dual-task performance. Foreman et al. in a recent (2013) report presented the results of a pilot study in which they examined patients with PD, as well as neurologically healthy participants during concurrent performance of postural and speaking tasks. Seven persons with PD and 7 healthy age-matched and 10 healthy young control subjects were tested in a motion capture facility. Task performances were performed concurrently and recorded during 3 time periods (acquisition (beginning and ending), 48-hour retention, and 1-week retention). In conclusion, the lack of consistent changes in motor performance in any of the tasks, except in the healthy young group, suggests a decreased efficiency of motor learning in the age-matched and PD groups and argues for increased practice dosages during balance training.

Table 1 contains the most commonly used test for assessment of balance and posture in Parkinson's disease. 
Table 1. Assessment of balance and posturein Parkinson`s disease.

\begin{tabular}{lll}
\hline Name & Abbrev. & Author/Year \\
\hline $\begin{array}{l}\text { Unified Parkinson`s Disease Rating } \\
\text { Scale part III }\end{array}$ & UPDRS & Fahn\& Elton, 1987 \\
\hline Timed Up and Go Test & TUG & $\begin{array}{l}\text { Podsiadło \& Richardson, } \\
1989\end{array}$ \\
\hline Berg Balance Scale & BBS & Berg, 1992 \\
\hline Tinetti Balance and Gait Assessment Tool & TBGAT & Tinetti, 1987 \\
\hline Brunel Balance Assessment & BBA & Tyson \& DeSouza, 2002 \\
\hline Functional Reach Test & FRT & Behrman et al.2002 \\
\hline Activity Specific Balance Confidence & ASBC & Powell 1995; Talley, 2008 \\
\hline Balance Evaluation Systems Test & BESTest & Horak et al., 2009 \\
\hline Balance Evaluation Systems Mini Test & MiniBESTest & $\begin{array}{l}\text { Franchignoni et al. 2012; } \\
\text { King \& Horak, 2013 }\end{array}$ \\
\hline
\end{tabular}

Arm and hand function. Among thev skill tests, the best known are the Purdue Pegboard Test, the Nine-Hole Peg Test (NHPT, Kellor et al., 1971), Jebsen and Taylor test, PigTail Test, Frenchay Arm Test (FAT), Action Research Arm Test (ARAT) and Wolf Motor Function Test (WFMT). Of the motricity scales, the most popular are the Fugl-Meyer Motor Assessment Scale (FMA, 1975) and Södring Motor Evaluation (SMES, 1994; Opara, 2012).

In 1990, Shimoyama et al. developed the Finger-Tapping Test (FTT). Subjects are asked to tap for $15 \mathrm{~s}$ at each subject's maximum tapping rate. Further studies have confirmed that FTT disease using low-cost accelerometers enables automatic prediction of MDS-UPDRS scores (Stamatakis et al., 2013), correlates with UPDRS Motor Disability and reveals improvement in fine motor control from medication and Deep Brain Stimulation (DBS)(Taylor et al., 2005).

Marusiak et al. concluded in 2009 that compared to controls, PD patients exhibited a higher amplitude in the biceps and triceps brachii muscles and lower median frequency of the mechanomyographical (MMG) signals in the both tested muscles. However, no differences in the EMG amplitude and an increase of the EMG median frequency in the triceps brachii muscle of the PD group were observed. The MMG was not affected by physiological postural tremor, and can depict differences between PD patients and controls, which may suggest that it is a valuable tool for neuromuscular assessment for this condition.

Fernandez-Del-Olmo et al. (2013) in their study described the investigations of electromyography pattern and the reaction time during a wrist flexion movement in response to three different stimuli: a visual imperative stimulus, visual stimulus simultaneous with a non-startle auditory stimulus, and with a startle auditory stimulus. Their results suggest that the startle reaction effect for upper limb movements are unimpaired in PD patients, and has different characteristics than the effect of non-startle stimuli.

Berardelli et al. (1986) studied rapid wrist flexion movements in a group of 10 patients with PD, compared with the same movements made by a group of 8 normal individuals. When the normal subjects made movements through 60 degrees, the first agonist burst of EMG activity in the wrist flexor muscles was longer and larger than that seen in movements of 15 degrees. They concluded that changes in the velocity of movements at a single joint are not a good reflection of the overall clinical state of patients with PD.
Table 2. Assessment of hand and arm function in Parkinson's disease

\begin{tabular}{lll}
\hline Name & Abbrev. & Author/Year \\
\hline $\begin{array}{l}\text { Unified Parkinson`s Disease Rating Scale } \\
\text { part II and III }\end{array}$ & UPDRS & Fahn\& Elton, 1987 \\
\hline Purdue Pegboard Test & PPT & Tiffin, 1948 \\
\hline Nine-Hole Peg Test & NHPT & Kellor et al.,1971 \\
\hline Jebsen and Taylor test & JTT & Jebsen \& Taylor, 1969, 1971 \\
\hline Pig-Tail Test & PTT & - (?) - \\
\hline Frenchay Arm Test & FAT & Wade et al.,1983 \\
\hline Action Research Arm Test & ARAT & Lyle, 1981; van der Lee, 2002 \\
\hline Wolf Motor Function Test & WFMT & Wolf et al., 1989, 1991 \\
\hline Fugl-Meyer Motor Assessment Scale & FMAA & Fugl-Meyer et al.,1975 \\
\hline Södring Motor Evaluation & SMES & Södring, 1994 \\
\hline Finger-Tapping Test & FTT & Shimoyama et al.,1990 \\
\hline
\end{tabular}

Table 2 contains the most commonly used test for assessment of hand and arm function in Parkinson's disease.

Walking in PD. According to Rochester et al. (2004), PD subjects walk at a significantly slower speed $(26.5 \%$; $\mathrm{P}<.001)$ and reduced step length $(23 \% ; \mathrm{P}<.001)$ than controls. Elbers et al. (2013) stated that timed walking tests are valid measurements to predict community walking in PD. However, evaluation of community walking should include an assessment of fear of falling.

The research team led by Hobart developed in 2003 a twelve-scale factors, 12-Item MS Walking Scale (MSWS-12) which is also useful in PD. Stina Bladh et al. (2012), according to a postal survey of $189 \mathrm{PD}$ subjects stated that the generic Walk-12G walking scale meets the rating scale criteria for clinical trials.

King et al. (2013) were interested in which outcome measures are sensitive to exercise intervention and explored the effects of two different exercise programmes for improving mobility in patients with PD. Thirty-nine participants were randomized into either the Agility Boot Camp (ABC) or treadmill training; $4 \mathrm{x} /$ week for 4 weeks. Four of five variables at the structure/function level changed after exercise: turn duration $(\mathrm{P}=0.03)$, stride velocity $(\mathrm{P}=0.001)$, peak arm speed $(\mathrm{P}=0,001)$, and horizontal trunk ROM during gait $(\mathrm{P}=0.02)$. Most measures improved similarly for both interventions. The only variable that detected a difference between groups was postural sway in $\mathrm{ABC}$ group $(\mathrm{F}=4.95$; $\mathrm{P}=0.03)$. The authors concluded that the outcome measures at ICF body structure/function level were most effective at detecting change after exercise, and revealing differences in improvement between interventions.

Recently, Klucken et al. (2013) developed a biosensorbased Embedded Gait Analysis using Intelligent Technology (eGaIT), which consist of accelerometers and gyroscopes attached to shoes that record motion signals during standardized gait and leg function. The results were confirmed in a second independent validation cohort (42 patients, 39 controls). eGaIT was able to successfully distinguish PD patients from controls with UPDRS-III classification rate of $81 \%$.

Table 3 contains the most commonly used tests for assessment of walking in Parkinson`s disease. 
Table 3. Assessment of walkingin Parkinson`s disease

\begin{tabular}{lll}
\hline Name & Abbrev. & Author/Year \\
\hline $\begin{array}{lll}\text { Unified Parkinson`s Disease Rating Scale part } \\
\text { II and III }\end{array}$ & UPDRS & Fahn \& Elton, 1987 \\
\hline Timed 10-Metre Walk Test & 10MT & Bohannon et al.,1996 \\
\hline Timed 20-Metre Walk Test & 20MT & Cunningham et al.1982 \\
\hline Two-Minute Walk Test & 2MWT & Butland et al., 1982 \\
\hline Six-Minute Walk Test & 6MWT & Balke, 1963 \\
\hline Functional Ambulation Category & FAC & Holden, 1984 \\
\hline Emory Functional Ambulation Profile & E-FAP & Wolff et al., 1999 \\
\hline
\end{tabular}

Three-dimensional motion assessment of gait in PD. Mirek et al. (2007) used three-dimensional analysis of movement which allowed precise assessment of gait disorders among 32 PD patients (age range: 50-75) in I-III stages of the disease according to Hoehn-Yarr. They concluded that patients with PD differed significantly from the control group in all spatiotemporal parameters; they had a clearly lower cadence, walking speed and step length, and their time for single and double support was longer. Among kinematic parameters of gait, patients with PD had a reduced range of movement in the lower limbs joints. They began the double support phase earlier and their swing phase was delayed when compared to controls.

The later stages of PD are characterized by altered gait patterns. Decreased arm swing has been described as an early sign of PD. Lewek et al. (2010) in 12 individuals with early PD (in 'off' state) and 8 controls, conducted gait analysis using an optically-based motion capture system. Twelve individuals with early PD (in 'off' state) and 8 controls underwent gait analysis using an optically-based motion capture system. Both groups had comparable gait velocities $(\mathrm{p}=0.61)$, and there was no significant difference between the groups in the magnitude of arm swing in all walking conditions for the arm that swung more $(p=0.907)$ or less $(p=0.080)$. Strikingly, the $\mathrm{PD}$ group showed significantly greater arm swing asymmetry (asymmetry angle: $13.9+$ or $-7.9 \%$ ), compared to the control group (asymmetry angle: $5.1+$ or $-4.0 \%$; $=0,003$ ). Unlike arm swing magnitude, arm swing asymmetry unequivocally differed between people with early PD and controls.

Morris et al. (2005) examined whether people with PD have a central amplitude regulation disorder using threedimensional (3-D) gait analyses to compare the effects of medication and attentional strategies on gait in $12 \mathrm{PD}$ subjects and 12 matched comparison subjects. They found evidence for a centrally-mediated amplitude regulation disorder in PD.

Recently, Speciali and al. (2013) published a study in which 14 patients with idiopathic PD and 9 healthy subjects participated. All subjects walked under 2 conditions: free walking and dual task (DT) walking. Then the Gait Deviation Index (GDI), developed by Schwartz and Rozumalski in 2008, were computed, the results showed gait impairment during DT, a significant difference between groups regarding GDI and an interaction effect involving the group, side and task factors. Both groups were different, independent of interference and side, but interference was only different for the PD group.

Tomlinson et al. in their recent review of techniques of physiotherapy used for Parkinson's disease (2014), stated the wide variety of outcome measures used, the most popular types of outcome measures of physiotherapy in PD are as following:

1) Gait outcomes such as: a. two- or six-minute walk test (Kersten 2004); b. walking speed: (i) 10- or 20-metre walk test (Kersten 2004), (ii). velocity in metres per second (Trew 2005); c. cadence in steps/min (Trew 2005); d. stride length in metres (Whittle 1996); e. step length (Trew 2005); f. Freezing of Gait Questionnaire (Giladi 2000).

2) Functional mobility and balance outcomes, such as: a. timed up and go (Podsiadło \& Richardson 1989), b. Functional Reach Test (Duncan 1990); c. Berg Balance Scale, (Berg 1992; Qutubuddin 2005); d. Activity Specific Balance Confidence (Powell 1995; Talley 2008).

3) Data on falls, such as: a. number of patients falling, e.g. falls diary; b. Falls Efficacy Scale, a 10-item patient-reported questionnaire better quality of life (Ware 1992).

\section{REFERENCES}

1. Berardelli A, Dick JP, Rothwell JC, Day BL, Marsden CD. Scaling of the size of the first agonist EMG burst during rapid wrist movements in patients with Parkinson's disease. J Neurol Neurosurg Psychiatry, 1986; 49(11): 1273-1279.

2. Behrman AL, Light KE, Flynn SM, Thigpen MT. Is the functional reach test useful for identifying falls risk among individuals with Parkinson's disease? Arch Physical Med Rehabil, 2002; 83(4): 538-542.

3. Bladh S, Nilsson MH, Hariz GM, Westergren A, Hobart J, Hagellet P. Psychometric performance of a generic walking scale (Walk-12G) in multiple sclerosis and Parkinson's disease. J Neurol, 2012; 259: 729-738.

4. Bloem BR, Grimbergen YA, Cramer M, Willemsen M, Zwinderman AH. Prospective assessment of falls in Parkinson's disease. J Neurol, 2001; 248(11): 950-958.

5. Błaszczyk JW, Orawiec R. Assessment of postural control in patients with Parkinson's disease: Sway ratio analysis. Human Movement Science, 2011; 30(2): 396-404.

6. Elbers RG, van Wegen EEH, Verhoef J, Kwakkel G. Is gait speed a valid measure to predict community ambulation in patients with Parkinson's disease? J Rehab Med, 2013, 45(4): 370-375.

7. Fahn S, Elton RL; Members of the UPDRS Development Committee. Unified Parkinson's Disease Rating Scale. in: Fahn S., Marsden CD, Calne D, Holstein N (eds.): Recent developments in Parkinson's disease. Vol. 2. Macmillian Healthcare Information, Plorhan Park, NJ, 1987: $153-163$.

8. Fernandez-Del-Olmo M, Bello O, Lopez-Alonso V, Marquez G, Sanchez JA, Morenilla L, Valls-Solé J. The effects of startle and non-startle auditory stimuli on wrist flexion movement in Parkinson's disease. Neurosci Lett, 2013; 548: 56-60.

9. Foreman KB, Sondrup S, Dromey C, Jarvis E, Nissen S, Dibble LE. The effects of practice on the concurrent performance of a speech and postural task in persons with Parkinson disease and healthy controls. Parkinsons Dis, 2013; 2013: 987621. doi: 10.1155/2013/987621. Epub 2013 Jun 11

10. Franchignoni F, Horak F, Godi M, Nardone A, Giordano A. Using psychometric techniques to improve the balance evaluation systems test: the mini-bestest. J Rehabil Med, 2010; 42(4): 323-331.

11. Gelb DJ, Oliver E, Gilman S. Diagnostic Criteria for Parkinson Disease. Arch Neurol 1999; 56(1): 33-39.

12. Goetz CG, Tilley BC, Shaftman SR, Stebbins GT, Fahn S, MartinezMartin P, Poewe W, Sampaio C, Stern MB, Dodel R, Dubois B, Holloway R, Jankovic J, Kulisevsky J, Lang AE, Lees A, Leurgans S, LeWitt PA, Nyenhuis D, Olanow CW, Rascol O, Schrag A, Teresi JA, van Hilten JJ, LaPelle N; Movement Disorder Society UPDRS Revision Task Force. Movement Disorder Society-sponsored revision of the Unified Parkinson's Disease Rating Scale (MDS-UPDRS): scale presentation and clinimetric testing results. Mov Disord, 2008; 23(15): 2129-2170.

13. Hobart JC, Riazi A, Lamping DL, Fitzpatrick R, Thompson AJ. Measuring the impact of MS on walking ability: the 12-Item MS Walking Scale (MSWS-12). Neurology, 2003; 60(1): 31-36.

14. Hoehn MM, Yahr MD. Parkinsonism: onset, progression and mortality. Neurol., 1967; 17: 427-442. 
15. Horak FB, Wrisley DM, Frank J. The Balance Evaluation Systems Test (BESTest) to Differentiate Balance Deficits. Phys Ther, 2009; 89(5): 484-498.

16. Hughes AJ, Daniel SE, Kilford L, Lees AJ. Accuracy of clinical diagnosis of idiopathic Parkinson's disease. A clinico-pathological study of 100 cases. JNNP, 1992; 55: 181-184.

17. King LA, Salarian A, Mancini M, Priest KC, Nutt J, Serdar A, Wilhelm J, Schlimgen J, Smith M, Horak FB. Exploring outcome measures for exercise intervention in people with Parkinson's disease. Parkinsons Dis, 2013; 2013: 572134. doi: 10.1155/2013/572134. Epub 2013 Apr 30.

18. Klucken J, Barth J, Kugler P, Schlachetzki J, Henze T, Marxreiter F, Kohl Z, Steidl R, Hornegger J, Eskofier B, Winkler J. Unbiased and mobile gait analysis detects motor impairment in Parkinson's disease. PLoS One, 2013; 8(2): e56956. doi: 10.1371/journal.pone.0056956. Epub 2013 Feb 19.

19. Lewek MD, Poole R, Johnson J, Halawa O, Huang X. Arm swing magnitude and asymmetry during gait in the early stages of Parkinson's disease. Gait Posture, 2010; 31(2): 256-260.

20. Marinus J, Visser M, Stiggelbout AM, Rabey JM, Martínez-Martín P, Bonuccelli U, Kraus PH, van Hilten JJ. A short scale for the assessment of motor impairments and disabilities in Parkinson's disease: the SPES/ SCOPA. J Neurol Neurosurg Psychiatry, 2004; 75: 388-395.

21. Marras C, Rochon P, Lang AE. Predicting motor decline and disability in Parkinson disease: a systematic review. Arch Neurol, 2002; 59(11): 1724-1728.

22. Martinez-Martin P, Visser M, Rodriguez-Blazquez C, Marinus J, Chaudhuri KR, van Hilten JJ; SCOPA-Propark Group; ELEP Group. SCOPA-sleep and PDSS: two scales for assessment of sleep disorder in Parkinson's disease. Mov Disord, 2008; 23(12): 1681-1688.

23. Marusiak J, Jaskólska A, Kisiel-Sajewicz K, Najwer W, Kisiel-Sajewicz K, Jaskólski A. EMG and MMG activities of agonist and antagonist muscles in Parkinson's disease patients during absolute submaximal load holding. J Electromyography Kinesiology, 2009; 19: 903-914.

24. Mirek E, Rudzińska M, Szczudlik A. Assessment of gait in patients with Parkinson's disease using three-dimensional motion analysis system Vicon . Neurol Neurochir Pol, 2007; 41(2): 128-133.

25. Morris M, Iansek R, McGinley J, Matyas T, Huxham F. Threedimensional gait biomechanics in Parkinson's disease: Evidence for a centrally mediated amplitude regulation disorder. Mov Disord, 2005; 20(1): 40-50.

26. Morris S, Morris ME, Iansek R. Reliability of measurements obtained with the Timed "Up \& Go" test in people with Parkinson disease. Phys Ther, 2001; 81(2): 810-818.

27. Opara J., Błaszczyk J., Dyszkiewicz A. Prevention of falls in Parkinson Disease. Medical Rehabil, 2005; 9(1): 31-34.

28. Opara J. Clinimetrics in neurorehabilitation. PZWL, Warszawa 2012 (in Polish).
29. Opara J. Clinimetrics in parkinsonism. Neurol Neurochir Pol, 1998; 6: 1497-1511 (in Polish).

30. Podsiadło D, Richardson S. The timed "Up and Go" test. Arch Phys Med Rehabil, 1989; 67: 387-389.

31. Rochester L, Hetherington V, Jones D, Nieuwboer A, Willems AM, Kwakkel G, Van Wegen E. Attending to the task: Interference effects of functional tasks on walking in Parkinson's disease and the roles of cognition, depression, fatigue, and balance. Arch Phys Med Rehabil, 2004: 85(10): 1578-1585.

32. Shimoyama I, Ninchoji T, Uemura K. The Finger-Tapping Test. A Quantitative Analysis. Arch Neurol, 1990; 47(6): 681-684.

33. Siggeirsdóttir K, Jónsson BY, Jónsson H Jr, Iwarsson S. The timed 'Up \& Go' is dependent on chair type. Clin Rehabil, 2002; 16(6): 609-616.

34. Słomka K, Juras G, Sobota G, Bacik B. The reliability of a ramblingtrembling analysis of center of pressure measures. Gait Posture, 2013; 37(2): 210-213.

35. Song J, Sigward S, Fisher B, Salem GJ. Altered Dynamic Postural Control during Step Turning in Persons with Early-Stage Parkinson's Disease. Hindawi Publishing Corp. Parkinson's Disease, 2012; 2012: $1-8$.

36. Speciali DS, de Oliveira EM, dos Santos NM, Pereira FV, Fracini AC, Fukuda TY, Oliveira CS, Correa JC, Lucareli PR. Use of the Gait Deviation Index and spatiotemporal variables for the assessment of dual task interference paradigm. J Bodyw Mov Ther, 2013; 17(1): 19-27.

37. Stamatakis J, Ambroise J, Crémers J, Sharei H, Delvaux V, Macq B, Garraux G. Finger tapping clinimetric score prediction in Parkinson's disease using low-cost accelerometers. Comput Intell Neurosci, 2013; 2013: 717853. doi: 10.1155/2013/717853. Epub 2013 Apr 16.

38. Stożek J, Rudzińska M, Longawa K, Szczudlik A. Effect of a comprehensive program rehabilitation on balance and gait in patients with Parkinson's disease. Neurol Neurochir Pol, 2003; supl. 5: 67-81.

39. Taylor Tavares AL, Jefferis GS, Koop M, Hill BC, Hastie T, Heit G, Bronte-Stewart HM. Quantitative Measurements of Alternating Finger Tapping in Parkinson's Disease Correlate With UPDRS Motor Disability and Reveal the Improvement in Fine Motor Control From Medication and Deep Brain Stimulation. Mov Disord. 2005; 20(10): 1286-1298.

40. Tinetti ME. Performance-oriented assessment of mobility problems in elderly patients. J Am Geriatr Soc, 1986; 34: 119-126.

41. van der Burg JC, van Wegen EE, Rietberg MB, Kwakkel G, van Dieën JH. et al. Postural control of the trunk during unstable sitting in Parkinson's disease. Parkinsonism Relat Disord, 2006; 12(8): 492-498.

42. Tomlinson CL, Herd CP, Clarke CE, Meek C, Patel S, Stowe R, Deane KHO, Shah L, Sackley CM, Wheatley K, Ives N. Physiotherapy for Parkinson's disease: a comparison of techniques (Review). Cochrane Database Syst Rev, 2014; 6: CD002815. 